KANAZAWA-05-08

April, 2005

\title{
Nonthermal production of baryon and dark matter
}

\author{
Daijiro Suematsu * \\ Institute for Theoretical Physics, Kanazawa University, \\ Kanazawa 920-1192, JAPAN
}

\begin{abstract}
We study nonthermal production of baryon and dark matter. If we extend the MSSM by introducing some singlet chiral superfields so as to enlarge the conserved global symmetry, the abundance of the baryon and the dark matter in the universe may be explained as the charge asymmetry of that symmetry. In such a case, the baryon energy density and the dark matter energy density in the present universe can be correlated each other and take the similar order values naturally.
\end{abstract}

*e-mail:suematsu@hep.s.kanazawa-u.ac.jp 
Both energy densities of baryon and dark matter in the universe have been measured through observations of the CMB anisotropy [1] and the large scale structure [2]. In the present astroparticle physics, it is one of crucial problems to explain why these energy densities take the similar order values. In the supersymmetric framework, the dark matter abundance is usually considered to be explained through the thermal production of the lightest stable superparticles with the weak interaction. Although it is considered to be a promising possibility, the scenario has a large dependence on the feature of supersymmetry (SUSY) breakings [3] and we also need an independent mechanism to produce the baryon number abundance. The SUSY breaking parameters seem to be required to be tuned to realize the observed abundances.

On the other hand, many years ago, an interesting idea was proposed in [4], where both abundances of the baryon and the dark matter are related to the charge asymmetry of the same global symmetry. The scenario seems to be able to explain naturally why they have the similar order values. Although the idea is very elegant, such a kind of realistic model seems not to have been constructed a lot in the context of SUSY models. ${ }^{1}$ In fact, since the Lagrangian of the minimal supersymmetric standard model (MSSM) has only baryon number $(B)$ and lepton number $(L)$ as its global symmetry, it may be difficult to fulfill the required condition to realize that scenario. ${ }^{2}$ However, if we introduce new singlet chiral superfields in the MSSM, the global symmetry can be extended and the idea may be applicable to the SUSY model.

In the MSSM, both operators $\hat{H}_{1} \hat{H}_{2}$ and $\hat{L} \hat{H}_{2}$ are gauge invariant where $\hat{L}$ and $\hat{H}_{1,2}$ are the lepton doublet and Higgs doublet chiral superfields. Thus we can construct two gauge invariant dimension three operators $\hat{S}_{1} \hat{H}_{1} \hat{H}_{2}$ and $\hat{N} \hat{L} \hat{H}_{2}$ by introducing singlet chiral superfields $\hat{S}_{1}$ and $\hat{N}$. If we add $\hat{S}_{1} \hat{H}_{1} \hat{H}_{2}$ to the MSSM superpotential and remove the ordinary $\mu$-term, we find that the global symmetry is extended. There appear two new Abelian symmetries other than the $B$ and $L$ symmetry $[7,8,9]$. They can be taken as the Peccei-Quinn symmetry and the $R$ symmetry. An example of their charge assignment for

\footnotetext{
${ }^{1}$ An example of this kind of works can be found in [5]. The production of baryons and dark matter through the nonthermal decay process has been also discussed in the MSSM context in [6].

${ }^{2}$ Although one might consider to use $L$ or $B-L$ as the global charge discussed in the following study, in that case we will face the difficulty such that it cannot explain the $B$ asymmetry and the small neutrino masses, simultaneously.
} 


\begin{tabular}{c|cccccccccccc} 
& $\tilde{g}, \tilde{W}, \tilde{B}$ & $\hat{Q}_{L}$ & $\hat{\bar{U}}_{L}$ & $\hat{\bar{D}}_{L}$ & $\hat{L}_{L}$ & $\hat{\bar{E}}_{L}$ & $\hat{H}_{1}$ & $\hat{H}_{2}$ & $\hat{S}_{1}$ & $\hat{N}$ & $A_{S U(3)}$ & $A_{S U(2)}$ \\
\hline$Q_{\mathrm{PQ}}$ & 0 & 0 & -2 & 1 & -1 & 2 & -1 & 2 & -1 & 0 & $-\frac{3}{2}$ & -1 \\
$Q_{R}$ & -1 & $-\frac{1}{6}$ & -1 & 0 & $-\frac{5}{6}$ & $\frac{2}{3}$ & $-\frac{5}{6}$ & $\frac{1}{6}$ & $-\frac{1}{3}$ & $-\frac{1}{2}$ & -5 & $-\frac{13}{3}$ \\
$B$ & 0 & $\frac{1}{3}$ & $-\frac{1}{3}$ & $-\frac{1}{3}$ & 0 & 0 & 0 & 0 & 0 & 0 & 0 & $\frac{3}{2}$ \\
$L$ & 0 & 0 & 0 & 0 & 1 & -1 & 0 & 0 & 0 & 0 & 0 & $\frac{3}{2}$ \\
\hline
\end{tabular}

Table Global U(1) charge assignment for the fermionic components and gauge anomaly.

the fermionic components of the chiral superfields is shown in Table. ${ }^{3}$ Among these four Abelian symmetries, two global symmetries $\mathrm{U}(1)_{B-L}$ and $\mathrm{U}(1)_{X}$ remain as those with no $\mathrm{SU}(3)$ and $\mathrm{SU}(2)$ gauge anomaly. ${ }^{4}$ In this note we study a possibility that this $X$ charge asymmetry produced through the nonthermal process plays a role as the origin of both abundances of the baryon and the dark matter.

We consider a model defined by a superpotential $W_{\text {MSSM }}+W_{1}$. $W_{\text {MSSM }}$ is the superpotential for the MSSM Yukawa interactions

$$
W_{\mathrm{MSSM}}=y_{U}^{\alpha \beta} \hat{Q}_{\alpha} \hat{\bar{U}}_{\beta} \hat{H}_{2}+y_{D}^{\alpha \beta} \hat{Q}_{\alpha} \hat{\bar{D}}_{\beta} \hat{H}_{1}+y_{E}^{\alpha \beta} \hat{L}_{\alpha} \hat{\bar{E}}_{\beta} \hat{H}_{1}
$$

We introduce two massless MSSM singlet chiral superfields $\hat{S}_{1}$ and $\hat{S}_{2}$ for the construction of an additional superpotential $W_{1}$. It plays an essential role in the present scenario. We assign the integer charge $q(>0)$ and $-p(<0)$ for the discrete symmetry $Z_{m}$ to $\hat{S}_{1}$ and $\hat{S}_{2}$, respectively. If the least common multiple for $p$ and $q$ is assumed to be $p q$ and $p+q=n(n<m)$, a superpotential constructed from the lowest order $Z_{m}$ invariant operators can be written as

$$
W_{1}=\lambda_{1} \hat{S}_{1} \hat{H}_{1} \hat{H}_{2}+\lambda_{2} \hat{S}_{2} \hat{F}_{1} \hat{F}_{2}+\frac{d}{M_{\mathrm{pl}}^{n-3}} \hat{S}_{1}^{p} \hat{S}_{2}^{q}
$$

where couplings $\lambda_{1,2}$ and $d$ are assumed to be real and $d=O(1)$. We suppose that chiral superfields $\hat{H}_{1,2}$ and $\hat{F}_{1,2}$ have a suitable $Z_{m}$ charge so as to make the first two terms in $W_{1}$ be $Z_{m}$ invariant. $\hat{F}_{1,2}$ are assumed to have no MSSM gauge interactions but have

\footnotetext{
${ }^{3}$ We should note that $\hat{N} \hat{L} \hat{H}_{2}$ violates the conservation of $L$ for the charge assignment given in Table. This operator is important for both the explanation of the small neutrino mass and the generation of the $B$ asymmetry. This point will be discussed later.

${ }^{4}$ It is easily checked that the $\mathrm{U}(1)_{X}$ charge can be represented as the linear combination of the four global Abelian charges as $3 X=B+L-10 Q_{\mathrm{PQ}}+3 Q_{R}$.
} 
the global charge so that $\hat{S}_{2} \hat{F}_{1} \hat{F}_{2}$ is invariant. They constitute a hidden sector which interacts with the fields in the visible sector only through the gravity. If we assign the chiral superfield $\hat{S}_{2}$ the global charges so as to be $X\left(\hat{S}_{2}\right)=x$ with $B=L=0$, SUSY breaking operators associated to the last term in $W_{1}$ may violate the $X$ conservation to give them the $X$ charge $\Delta X=4 p+x q(\neq 0)$. We will show that it can generate the $X$ asymmetry through the AD mechanism [10]. In the following part, the inflaton is assumed to couple directly with the fields in the visible sector alone.

Now we consider a $D$-flat direction defined by $\left\langle S_{1}\right\rangle=\frac{u}{\sqrt{q}} e^{i \theta_{1}}$ and $\left\langle S_{2}\right\rangle=\frac{u}{\sqrt{p}} e^{i \theta_{2}}{ }^{5}$. This direction is slightly lifted by the nonrenormalizable operator in the scalar potential, which is induced from the last term of $W_{1}$. In the early universe, there are additional effective contributions to the scalar potential induced by the SUSY breaking effects caused by the large Hubble constant $H$ [11] and the thermal effects [12] other than the ordinary soft SUSY breakings. If we take account of these effects, the scalar potential in that direction is found to be expressed as

$$
\begin{aligned}
V & \simeq\left(-c H^{2}+M_{u}^{2}(T)\right) u^{2}+\frac{n|d|^{2}}{p^{q-1} q^{p-1}} \frac{u^{2 n-2}}{M_{\mathrm{pl}}^{2 n-6}} \\
& +\frac{1}{p^{\frac{q}{2}} q^{\frac{p}{2}}}\left\{\left(\frac{a m_{3 / 2} e^{i \theta_{a}}}{M_{\mathrm{pl}}^{n-3}}+\frac{b H e^{i \theta_{b}}}{M_{\mathrm{pl}}^{n-3}}\right) u^{n} e^{i n \theta}+\text { h.c. }\right\}
\end{aligned}
$$

where $\theta=\left(p \theta_{1}+q \theta_{2}\right) / n$. $m_{3 / 2}$ is a typical soft SUSY breaking scale of $O(1) \mathrm{TeV}$ and the coefficients $a, b$ and $c$ are $O(1)$ real constants. CP phases $\theta_{a}$ and $\theta_{b}$ in the curly brackets are induced by the above mentioned SUSY breaking effects which violate the $\mathrm{U}(1)_{X}$ by an amount of $\Delta X$. The effective mass $M_{u}^{2}(T)$ contains the usual soft SUSY breaking mass $m_{S}^{2}$ of $O\left(m_{3 / 2}^{2}\right)$ and the thermal mass $C_{T} \lambda_{1}^{2} T^{2}$ caused by the coupling of $\hat{S}_{1}$ with $\hat{H}_{1,2}$ in the thermal plasma. ${ }^{6}$ It can be expressed depending on the value of $u$ as [12]

$$
M_{u}^{2}(T) \simeq \begin{cases}m_{S}^{2} & \left(\lambda_{1} u>T\right), \\ m_{S}^{2}+C_{T} \lambda_{1}^{2} T^{2} & \left(\lambda_{1} u<T\right),\end{cases}
$$

where $C_{T}$ is a numerical factor in the thermal mass.

In the scalar potential (3), the Hubble constant contribution $H^{2}$ dominates the mass of the condensate during the inflation. If the sign of this Hubble constant contribution is

\footnotetext{
${ }^{5}$ We note that the potential minimum can be realized along the subspace $q\left|S_{1}\right|^{2}=p\left|S_{2}\right|^{2}$ as far as the soft SUSY breaking masses of $S_{1}$ and $S_{2}$ are equal.

${ }^{6}$ Since the inflaton is assumed not to couple directly with the hidden sector fields, no thermal plasma appears in the hidden sector.
} 
negative $(c>0)[11]$, the magnitude of the condensate takes a large value such as

$$
u_{I} \simeq\left(H M_{\mathrm{pl}}^{n-3}\right)^{\frac{1}{n-2}}
$$

On the other hand, the phase $\theta$ of the condensate at the potential minimum takes one of the $n$ distinct values $\theta=-\theta_{b} / n+2 \pi \ell / n(\ell=1,2, \cdots, n)$. At this period the condensate follows this instantaneous potential minimum since its evolution is almost the critical damping. The dilute plasma appears as a result of a partial decay of the inflaton. Then the temperature rapidly increases to $T_{\max } \simeq\left(T_{R}^{2} H M_{\mathrm{pl}}\right)^{1 / 4}[12] . T_{R}$ is the reheating temperature realized after the completion of the inflaton decay and can be expressed as $T_{R} \simeq \sqrt{M_{\mathrm{pl}} \Gamma_{I}}$ where $\Gamma_{I}$ is the inflaton decay width. If this temperature $T_{\max }$ does not satisfy $\lambda_{1}\left|u_{I}\right|<T_{\max }$, no thermal contribution to $M_{u}^{2}(T)$ appears and $M_{u}^{2}(T)$ takes the expression of the upper one in eq. (4) $[11,12]$. Thus, the condition for the thermal effects to be negligible during the inflation gives the following lower bound on $\lambda_{1}$ :

$$
\lambda_{1}>T_{R}^{1 / 2} H_{I}^{\frac{n-6}{4(n-2)}} M_{\mathrm{pl}}^{\frac{10-3 n}{4(n-2)}}
$$

where $H_{I}$ is the Hubble parameter during the inflation.

When the inflaton evolves and $H$ decreases to $H \sim m_{3 / 2},{ }^{7}$ the effective squared mass of the condensate becomes positive and then $u=0$ is the minimum of the scalar potential $V$. The condensate starts to oscillate around $u=0$, and the thermal effects due to the dilute plasma to $M_{u}^{2}(T)$ is expected to appear. Then $M_{u}^{2}(T)$ takes the expression of the lower one in eq. (4). At this time, the dominant term for the $\mathrm{U}(1)_{X}$ breaking changes from the second term in the curly brackets of eq. (3) to the first one. Since the phase $\theta_{a}$ and $\theta_{b}$ are generally independent, the phase $\theta$ of the condensate changes non-adiabatically from that determined by $\theta_{b}$ to that determined by $\theta_{a}$ due to the torque in the angular direction. Thus, the $X$ asymmetry is stored in the condensate during its evolution due to the AD mechanism [10].

The produced $X$ asymmetry can be estimated by taking account that the $X$ current conservation is violated by the dominant $X$ breaking operator in the curly brackets in eq. (3) as

$$
\frac{d \Delta n_{X}(t)}{d t}=\Delta X \frac{a m_{3 / 2} u^{n}}{M_{\mathrm{pl}}^{n-3}} \sin \delta,
$$

\footnotetext{
${ }^{7}$ Here we assume an inflation scenario in which this period is before the reheating. This means that $m_{3 / 2}>\Gamma_{I}$ is satisfied and then $T_{R}<\sqrt{M_{\mathrm{pl}} m_{3 / 2}} \simeq 10^{11} \mathrm{GeV}$.
} 
where $\Delta X$ is the $X$ charge of that operator and $\delta$ is determined by the difference of $\theta_{a}$ and $\theta_{b}$. By solving this equation, the $X$ asymmetry produced in the condensates $\left\langle S_{1,2}\right\rangle$ at this period is found to be roughly expressed as $[10,11,12]^{8}$

$$
\Delta n_{X}(t) \simeq \Delta X \frac{m_{3 / 2}}{H} H^{\frac{n}{n-2}} M_{\mathrm{pl}}^{\frac{2(n-3)}{n-2}} \sin \delta
$$

where $t$ is the time when $H \sim m_{3 / 2}$. Following this, the reheating due to the inflaton decay is completed at $H \sim \Gamma_{I}$.

The $X$ asymmetry stored in the condensate is liberated into the thermal plasma in the visible sector and also into the hidden sector through the decay of the condensate by the $X$ conserving couplings $\lambda_{1} \hat{S}_{1} \hat{H}_{1} \hat{H}_{2}$ and $\lambda_{2} \hat{S}_{2} \hat{F}_{1} \hat{F}_{2}$, respectively. We assume that the decay widths through these couplings satisfy $\Gamma_{S_{1}} \simeq \Gamma_{S_{2}}$ to make the discussion clear. ${ }^{9}$ Since the oscillation of the condensate behaves as a matter for the expansion of the universe, it can dominate the energy density of the universe before its decay which occurs at $H \sim \Gamma_{S_{1,2}}$. For the reasonable value of $\lambda_{1,2}$, this is the case since $\Gamma_{S_{1,2}}<\Gamma_{I}$ is satisfied. Taking account of this, the ratio of the $X$ asymmetry $\Delta n_{X}^{V, H}$ liberated into each sector to the entropy density $s$ is estimated as

$$
Y_{X}^{V, H} \equiv \frac{\Delta n_{X}^{V, H}\left(\tilde{t}_{R}\right)}{s}=\frac{\Delta n_{X}\left(\tilde{t}_{R}\right)}{2 s} \simeq \frac{\Delta n_{X}(t)}{2 \tilde{T}_{R}^{3}} \frac{t^{2}}{\tilde{t}_{R}^{2}} \simeq \frac{\Delta X}{2} \tilde{T}_{R} m_{3 / 2}^{\frac{4-n}{n-2}} M_{\mathrm{pl}}^{\frac{-2}{n-2}} \sin \delta,
$$

where we use $\tilde{t}_{R} \sim \Gamma_{S_{1,2}}^{-1} \sim M_{\mathrm{pl}} / \tilde{T}_{R}^{2}$. If the temperature $\tilde{T}_{R}\left(\simeq 10^{10} \lambda_{1} \mathrm{GeV}\right)$ is appropriate to keep the $X$ asymmetry ${ }^{10}$ and convert it into the $B$ asymmetry through the sphaleron interaction [14], we can obtain the $B$ asymmetry in the visible sector [9]. The $X$ asymmetry liberated in the hidden sector is considered to explain the dark matter as far as the $X$ charge is conserved in the hidden sector.

We examine whether the $X$ asymmetry in the visible sector transformed into the thermal plasma through the decay of the condensate can remain as a nonzero value and be partially converted into the $B$ asymmetry. This should be studied taking account that the electroweak sphaleron interaction and other various interactions are in the thermal equilibrium. For this study, it is convenient to consider the detailed balance of these interactions and solve the chemical equilibrium equations $[15,16]$. The particle-antiparticle

\footnotetext{
${ }^{8}$ The rigorous estimation requires the numerical calculation as discussed in [11]. It is beyond the scope of this paper and we do not go further here.

${ }^{9}$ General cases will be discussed in Appendix.

${ }^{10}$ This $\tilde{T}_{R}$ is found to be a marginal value for the cosmological gravitino problem [13].
} 
number asymmetry $\Delta n_{f}$ can be approximately related to the corresponding chemical potential $\mu_{f}$. In the case of $\mu_{f} \ll T$, it can be represented as

$$
\Delta n_{f} \equiv n_{f}-n_{f^{c}}= \begin{cases}\frac{g_{f}}{6} T^{2} \mu_{f} & (f: \text { fermion }) \\ \frac{g_{f}}{3} T^{2} \mu_{f} & (f: \text { boson })\end{cases}
$$

where $g_{f}$ is a number of relevant internal degrees of freedom of the field $f$. By solving the detailed valance equations for the chemical potential $\mu_{f}$, we can estimate the charge asymmetry at the period after the decay of the condensate.

If the $\mathrm{SU}(2)$ and $\mathrm{SU}(3)$ sphaleron interactions are in the thermal equilibrium, we have the conditions such as

$$
\begin{aligned}
& \sum_{i=1}^{N_{g}}\left(3 \mu_{Q_{i}}+\mu_{L_{i}}\right)+\mu_{\tilde{H}_{1}}+\mu_{\tilde{H}_{2}}+4 \mu_{\tilde{W}}=0, \\
& \sum_{i=1}^{N_{g}}\left(2 \mu_{Q_{i}}-\mu_{U_{i}}-\mu_{D_{i}}\right)+6 \mu_{\tilde{g}}=0
\end{aligned}
$$

where $N_{g}$ is a number of the generation of quarks and leptons. The cancellation of the total hypercharge or the electric charge of plasma in the universe requires

$$
\begin{aligned}
& \sum_{i=1}^{N_{g}}\left(\mu_{Q_{i}}+2 \mu_{U_{i}}-\mu_{D_{i}}-\mu_{L_{i}}-\mu_{E_{i}}\right)+\mu_{\tilde{H}_{2}}-\mu_{\tilde{H}_{1}} \\
& \quad+2 \sum_{i=1}^{N}\left(\mu_{\tilde{Q}_{i}}+2 \mu_{\tilde{U}_{i}}-\mu_{\tilde{D}_{i}}-\mu_{\tilde{L}_{i}}-\mu_{\tilde{E}_{i}}\right)+2\left(\mu_{H_{2}}-\mu_{H_{1}}\right)=0 .
\end{aligned}
$$

When Yukawa interactions in $W_{\text {MSSM }}+W_{1}$ are in the thermal equilibrium, they impose the conditions ${ }^{11}$

$$
\begin{array}{ll}
\mu_{Q_{i}}-\mu_{U_{j}}+\mu_{H_{2}}=0, & \mu_{Q_{i}}-\mu_{D_{j}}+\mu_{H_{1}}=0, \\
\mu_{L_{i}}-\mu_{E_{j}}+\mu_{H_{1}}=0, & \mu_{S_{1}}+\mu_{\tilde{H}_{1}}+\mu_{\tilde{H}_{2}}=0 .
\end{array}
$$

There are also the conditions for the gauge interactions in the thermal equilibrium, which are summarized as

$$
\mu_{\tilde{Q}_{i}}=\mu_{\tilde{g}}+\mu_{Q_{i}}=\mu_{\tilde{W}}+\mu_{Q_{i}}=\mu_{\tilde{B}}+\mu_{Q_{i}}
$$

where $\mu_{\tilde{g}}, \mu_{\tilde{W}}$ and $\mu_{\tilde{B}}$ stand for gauginos in the MSSM. The similar relations to eq. (15) is satisfied for leptons $\hat{L}_{i}$, Higgs fields $\hat{H}_{1,2}$ and other fields $\hat{U}_{i}, \hat{D}_{i}, \hat{E}_{i}$ which have the SM

\footnotetext{
${ }^{11}$ We should note that the last term in $W_{1}$ leaves the thermal equilibrium at $T \sim M_{\mathrm{pl}}$. Since $\hat{S}_{1}$ has no other coupling to the MSSM contents than $\lambda_{1} \hat{S}_{1} \hat{H}_{1} \hat{H}_{2}$, the last one in eq. (14) is the only condition for $\mu_{S_{1}}$.
} 
gauge interactions. Flavor mixings of quarks and leptons due to the Yukawa couplings allow us to consider the flavor independent chemical potential such as $\mu_{Q}=\mu_{Q_{i}}$ and $\mu_{L}=\mu_{L_{i}}$.

Here we introduce an operator violating both $B-L$ and $X$, which is necessary to convert the $X$ asymmetry into the $B$ and $L$ asymmetry. If such an operator exists, only a linear combination of these two U(1)s is absolutely conserved. Then a part of $X$ asymmetry can be converted into the $B-L$ asymmetry. We consider an effective operator $\left(\hat{L} \hat{H}_{2}\right)^{2}$ as such an example. It corresponds to the effective neutrino mass operator in the ordinary seesaw mechanism, which is obtained from the operator $\hat{N} \hat{L} \hat{H}_{2}$ discussed in the first part by integrating out the heavy right-handed neutrino $\hat{N}$. The thermal equilibrium condition of this operator can be written as

$$
\mu_{L}+\mu_{H_{2}}=0
$$

By now we have not taken account of the equilibrium conditions for soft SUSY breaking operators. The soft SUSY breaking operators are in the thermal equilibrium when $H \underset{\sim}{<} \Gamma_{s s}$ is satisfied. Since the rate of the soft SUSY breaking effects is written as $\Gamma_{s s} \simeq m_{3 / 2}^{2} / T$ [7], we find that the soft SUSY breaking operators are in the thermal equilibrium for the temperature $T \lesssim T_{s s} \simeq 10^{7} \mathrm{GeV}$. Thus, for $T \lesssim T_{s s}$ we find that $\mu_{\tilde{g}}=0$ is satisfied and then eqs. $(11) \sim(16)$ result in $\mu_{\tilde{H}_{2}}=0$. The $X$ asymmetry produced in the visible sector through the decay of the condensate disappears in this case. In order to escape this, if we define $T_{X}$ as a temperature at which the $X$ and $B-L$ violating interaction is out-ofequilibrium, we need to require that $T_{X}$ should satisfy $T_{s s} \lesssim T_{X} \lesssim \tilde{T}_{R}$. In order that this condition is satisfied, the effective operator $\left(\hat{L} \hat{H}_{2}\right)^{2}$ have to leave the thermal equilibrium before the temperature reaches $T_{s s}$. By using the right-handed neutrino mass $M_{R}$, we can summarize this condition into a statement that $H>T_{X}^{3} / M_{R}^{2}$ should be satisfied at $T_{X} \gtrsim T_{s s}$. This results in $M_{R} \gtrsim 10^{12} \mathrm{GeV}$, which is a suitable value for the explanation of the light neutrino masses required by the neutrino oscillation data $[17,18]$. In that case we find that there is an independent chemical potential in these thermal equilibrium conditions $(11) \sim(16)$. It can be taken as $\mu_{\tilde{H}_{2}}$, which corresponds to the above mentioned remaining symmetry.

The $X$ asymmetry induced in the visible sector through the decay of the condensate can be partially converted into the $B$ asymmetry. By solving eqs. $(11) \sim(16), \mu_{Q}, \mu_{L}$, $\mu_{H_{1,2}}$ and $\mu_{\tilde{g}}$ can be written with the chemical potential of Higgsino field $\tilde{H}_{2}$ at $T_{X}$ in such 
a way as

$$
\begin{aligned}
& \mu_{Q}=\frac{17 N_{g}+6}{N_{g}\left(10 N_{g}^{2}-17 N_{g}-15\right)} \mu_{\tilde{H}_{2}}, \quad \mu_{L}=-\mu_{H_{2}}=\frac{5\left(4 N_{g}+3\right)}{10 N_{g}^{2}-17 N_{g}-15} \mu_{\tilde{H}_{2}}, \\
& \mu_{H_{1}}=-\frac{40 N_{g}+3}{10 N_{g}^{2}-17 N_{g}-15} \mu_{\tilde{H}_{2}}, \quad \mu_{\tilde{g}}=-\frac{\left(10 N_{g}+3\right) N_{g}}{10 N_{g}^{2}-17 N_{g}-15} \mu_{\tilde{H}_{2}} .
\end{aligned}
$$

Defining $B$ and $L$ as $\Delta n_{B} \equiv B T^{2} / 6$ and $\Delta n_{L} \equiv L T^{2} / 6$, we can calculate these values at $T_{s s}$ by using eqs. (10) and (17) as

$$
\begin{aligned}
& B=\frac{80 N_{g}^{3}+204 N_{g}^{2}-150 N_{g}-72}{360 N_{g}^{3}+3308 N_{g}^{2}-1419 N_{g}-1143} X_{1}, \\
& L=\frac{N_{g}\left(60 N_{g}^{2}-42 N_{g}-126\right)}{360 N_{g}^{3}+3308 N_{g}^{2}-1419 N_{g}-1143} X_{1},
\end{aligned}
$$

where $X_{1}$ stands for the $X$ asymmetry stored in the $S_{1}$ condensate, which is defined by $\Delta n_{X}^{V} \equiv X_{1} T^{2} / 6$. These results show that all of $B, L$ and $B-L$ take nonzero values as far as $X_{1} \neq 0$.

When the temperature goes below $T_{s s}$, the soft SUSY breaking operators are in the thermal equilibrium. As mentioned above, this results in $\mu_{\tilde{g}}=0$ and $X_{1}=0$. However, if the $X$ and $B-L$ violating interaction in the visible sector is assumed to be out-ofequilibrium at $T_{s s}$, the equilibrium conditions are represented by $(11) \sim(15)$. Thus the $B-L$ asymmetry existing at $T_{s s}$ is kept after this period. The equilibrium conditions give the ordinary MSSM values for $B$ and $L$ as

$$
B=\frac{4\left(2 N_{g}+1\right)}{22 N_{g}+13}(B-L), \quad L=-\frac{14 N_{g}+9}{22 N_{g}+13}(B-L),
$$

where we should use the $B-L$ value obtained from eq. (18). The $B$ asymmetry produced in this scenario is finally estimated as

$$
Y_{B} \equiv \frac{\Delta n_{B}}{s} \simeq \frac{\Delta n_{X}}{2 s} f\left(N_{g}\right) \kappa \simeq \frac{\Delta X}{2} \tilde{T}_{R} m_{3 / 2}^{\frac{4-n}{n-2}} M_{\mathrm{pl}}^{\frac{-2}{n-2}} f\left(N_{g}\right) \kappa \sin \delta
$$

where eq. (9) is used and $\kappa(\leq 1)$ is introduced to take account of the washout effect. $f\left(N_{g}\right)$ is a numerical factor defined by

$$
f\left(N_{g}\right)=\frac{B-L}{X_{1}} \frac{4\left(2 N_{g}+1\right)}{22 N_{g}+13}
$$

and it takes $f(3) \simeq 0.3$ for $N_{g}=3$. From this result, we find that this scenario can produce the presently observed $B$ asymmetry $Y_{B}=(0.6-1) \times 10^{-10}$ as far as $n \geq 5$ and in the case of $n=5$, for example, $\tilde{T}_{R} \gtrsim 10^{4} /(\Delta X \kappa \sin \delta) \mathrm{GeV}$ is required. 
Since the $X$ asymmetry in the hidden sector is expected to explain the dark matter, we can estimate the ratio of the baryon energy density to the dark matter energy density by using eqs. (9) and (20) in such a way as

$$
\frac{\Omega_{B}}{\Omega_{\mathrm{DM}}} \simeq \frac{m_{p} Y_{B}}{m_{\mathrm{LP}} Y_{X}^{H}} \simeq f(3) \kappa \frac{m_{p}}{m_{\mathrm{LP}}},
$$

where $\Omega_{i}$ is the ratio of the energy density $\rho_{i}$ to the critical energy density $\rho_{\text {cr }}$ in the universe. Masses of the proton and the lightest stable particles in the hidden sector with nonzero $X$ charge are represented by $m_{p}$ and $m_{\mathrm{LP}}$. This relation suggests that the presently observed value $\Omega_{B} / \Omega_{\mathrm{CDM}} \sim 0.17$ can be explained if $m_{\mathrm{LP}}$ is the same order value as $m_{p}$ in the case of $\kappa \simeq 1{ }^{12}$ This value of $m_{\mathrm{LP}}$ also suggests that this dark matter candidate behaves as the cold dark matter.

Finally we order some remarks. Firstly, the evolution of $u$ may be able to induce the $\mu$-term [9]. We assume $H_{I} \sim 10^{13} \mathrm{GeV}$ during the inflation on the basis of the CMB data and $T_{R} \lesssim 10^{9} \mathrm{GeV}$. For these values we obtain $T_{\max } \sim 10^{13} \mathrm{GeV}$. Thus, if we take $n=5 \mathrm{in}$ $W_{1}$, as an example, eq. (5) gives $u_{I} \sim 10^{16} \mathrm{GeV}$ and eq. (6) suggests that $\lambda_{1} \gtrsim 10^{-9} T_{R}^{1 / 2}$ should be satisfied. When the temperature decreases from $T_{\max }$ to $T_{c} \sim m_{3 / 2} / \lambda_{1}, M_{S_{1}}^{2}(T)$ represented by the lower one in eq. (4) starts to be dominated by the soft SUSY breaking mass $m_{S_{1}}^{2}$. If $m_{S_{1}}^{2}<0$ is realized by some reason [21], $u \neq 0$ becomes the true vacuum after this period. Since the $\mu$-term is generated from the first term in $W_{1}$ as $\mu=\lambda_{1} u$, such a value of $u$ should be $u_{0} \lesssim 10^{11} T_{R}^{-1 / 2} \mathrm{GeV}$ to realize the appropriate $\mu$ for the above mentioned $\lambda_{1} \cdot{ }^{13}$ Although the condensate again starts to oscillate around $u_{0}$, the deviation from $u_{0}$ instantaneously decays into the light fields through the $X$ conserving coupling $\hat{S}_{1} \hat{H}_{1} \hat{H}_{2}$ since $H<\Gamma_{S_{1}}$ is satisfied at this time. The released energy cannot dominate the total energy density $\left(\frac{\pi^{2}}{30} g_{*} T^{4} \gg m_{3 / 2}^{2} u_{0}^{2}\right)$ and then the effects of the produced entropy is negligible. Thus, even in this case the $X$ asymmetry obtained in eq. (9) can be used as the origin of the $B$ asymmetry.

Secondly, it is useful to show a simple example of the mass generation scenario to make the picture of the hidden sector a little bit clear, although there seem to be various

\footnotetext{
${ }^{12}$ This tuning of the mass of the dark matter field seems to be generally required and cannot be avoided even in the case that both number densities are related. An example evading this can be found in [19] where the QCD-balls play the role of the dark matter.

${ }^{13} \mathrm{Such}$ a $u_{0}$ may be expected to be determined either by the nonrenormalizable terms or by the pure radiative symmetry breaking effect in which $u_{0}$ is estimated by using the renormalization group equation [22].
} 
possibilities of the structure for the hidden sector which can realize the right value for $m_{\mathrm{LP}}$ within the present framework. ${ }^{14}$ We consider the hidden sector composed of new chiral superfields $\hat{C}_{1,2}$ and $\hat{S}$ in addition to $\hat{F}_{1,2}$ and $\hat{S}_{2}$ in $W_{1}$. If we suppose the $X$ charge assignment for these chiral superfields such as

$$
\begin{aligned}
& X\left(\hat{F}_{1}\right)=1-x, \quad X\left(\hat{F}_{2}\right)=1, \quad X\left(\hat{C}_{1}\right)=1+x, \\
& X\left(\hat{C}_{2}\right)=2-x, \quad X(\hat{S})=0, \quad X\left(\hat{S}_{2}\right)=x
\end{aligned}
$$

we can have the supplementary superpotential for these chiral superfields as

$$
W_{2}=h_{1} \frac{\hat{S}^{2}}{M_{\mathrm{pl}}} \hat{F}_{1} \hat{C}_{1}+h_{2} \hat{S} \hat{S}_{2} \hat{C}_{2}+\frac{h_{3}}{2} \hat{S} \hat{F}_{2}^{2}
$$

We find that these operators are the lowest order ones allowed for the above $X$ charge assignment. $^{15}$ If $\langle S\rangle$ takes a nonzero value, fermions in the hidden sector get masses through the interactions in $W_{2}$ without breaking the $X$ charge. Their mass terms can be written as

$$
\left(C_{1}, F_{1}\right)\left(\begin{array}{cc}
0 & h_{1} \frac{\langle S\rangle^{2}}{M_{\mathrm{pl}}} \\
h_{1} \frac{\langle S\rangle^{2}}{M_{\mathrm{p} 1}} & 0
\end{array}\right)\left(\begin{array}{c}
C_{1} \\
F_{1}
\end{array}\right)+\left(C_{2}, S_{2}\right)\left(\begin{array}{cc}
0 & h_{2}\langle S\rangle \\
h_{2}\langle S\rangle & 0
\end{array}\right)\left(\begin{array}{c}
C_{2} \\
S_{2}
\end{array}\right)+h_{3}\langle S\rangle F_{2}^{2} .
$$

where $\left\langle S_{2}\right\rangle$ is assumed to be zero since it breaks the $X$ charge. From this, we find that the lightest mass eigenvalue is $m_{\mathrm{LP}} \simeq h_{1}\langle S\rangle^{2} / M_{\mathrm{pl}}$. This $m_{\mathrm{LP}}$ can be in a required region as far as $\langle S\rangle=O\left(10^{9}\right) \mathrm{GeV}$ is realized. Although the value of $\langle S\rangle$ depends on the more detailed structure of the hidden sector, we can expect that it will occur in the similar way discussed in [22]. The $X$ charge asymmetry distributed into the fermionic component of $\hat{F}_{1}$ through the coupling $\lambda_{2} \hat{S}_{2} \hat{F}_{1} \hat{F}_{2}$ can present a suitable amount of the energy density of dark matter. ${ }^{16}$

Thirdly, we remark the relation to the ordinary leptogenesis. In the seesaw model the leptogenesis is usually considered on the basis of the out-of-equilibrium decay of the heavy right-handed neutrinos [23] or the decay of sneutrino condensate [24]. However,

\footnotetext{
${ }^{14}$ We will present another example in the extended version prepared in [20].

${ }^{15}$ It should be noted that the lower dimensional $X$ invariant renormalizable operators $\hat{S}_{2} \hat{C}_{2}, \hat{F}_{2}^{2}$ and $\hat{S} \hat{F}_{1} \hat{C}$ can be forbidden by imposing the $Z_{m}$ symmetry appropriately.

${ }^{16}$ Although the fermionic component of $\hat{S}$ is massless in the present example, $\hat{F}_{1}$ has no renormalizable interaction with $\hat{S}$ and there is no effective decay mode between them. Thus, the $X$ charge asymmetry stored in the fermionic component of $\hat{F}_{1}$ is considered to be conserved.
} 
if we consider the spontaneous $\mu$-term generation along the almost flat direction of $\left\langle S_{1}\right\rangle$ as discussed above, the $B$ asymmetry produced by this usual leptogenesis might not be the dominant one. As mentioned before, we assume that the decay of the condensate is completed above the temperature $T_{X}$ which can be sufficiently lower than the masses of the right-handed heavy neutrinos. Then the $B$ asymmetry produced through the usual scenario seems to be washed out or overridden by the $B$ asymmetry produced in the present scenario.

Fourthly, we refer to the experimental signatures of the present model. Since the dark matter field lives in the hidden sector in the present model, it interacts with the fields in the observable sector only through the gravitational interaction. This may make it discriminate experimentally from other candidates in the MSSM. In the visible sector, we can also find the experimental signatures of this model in the the neutral Higgs and neutralino sectors. Since this model is extended from the MSSM by the singlet chiral superfield $\hat{S}_{1}$ which has a coupling $\lambda_{1} \hat{S}_{1} \hat{H}_{1} \hat{H}_{2}$, the neutral Higgs and neutralino sectors are changed from the MSSM. In the neutral Higgs scalar, there is an additional contribution for the neutral Higgs mass from this coupling. Thus the lightest neutral Higgs scalar can be heavier than that in the MSSM. In the neutralino sector also, some of the neutralinos can have an substantial ingredient coming from the fermionic component of $\hat{S}_{1}$. If we combine these features of the model, it may be possible to distinguish the present model from other proposals in the MSSM.

In summary, we have studied the possibility that both abundances of the baryon and the dark matter are originated from the asymmetry of the same global charge in the supersymmetric framework. In order to enlarge the global symmetry of the MSSM, we have introduced some singlet chiral superfields. In that model we have shown that both observed values of $Y_{B}$ and $\Omega_{B} / \Omega_{\mathrm{CDM}}$ could be realized through the global charge asymmetry stored in the condensate of these singlet scalar components. This kind of possibility for the production of the $B$ asymmetry and the dark matter may be worth to further study as much as the usual scenario.

This work is supported in part by a Grant-in-Aid for Scientific Research (C) from Japan 
Society for Promotion of Science (No. 14540251).

\section{Appendix}

In this appendix we discuss the amount of $X$ asymmetry liberated into each sector in general cases with arbitrary $\Gamma_{S_{1}}$ and $\Gamma_{S_{2}}$. If we take account that the decay products of the condensate behave as the radiation and also no photon is produced through its decay into the hidden sector, we can estimate the $X$ asymmetry $\Delta n_{X}^{V}\left(\tilde{t}_{R}\right)$ generated in the visible sector in case $\Gamma_{S_{1}}>\Gamma_{S_{2}}$ as,

$$
\frac{\Delta n_{X}^{V}\left(\tilde{t}_{R}\right)}{s}=\frac{\Delta n_{X}(t)}{s} \frac{\Gamma_{S_{1}}}{\Gamma_{S_{1}}+\Gamma_{S_{2}}}\left(\frac{t}{t_{1}}\right)^{2}
$$

and also in case $\Gamma_{S_{1}}<\Gamma_{S_{2}}$ as,

$$
\frac{\Delta n_{X}^{V}\left(\tilde{t}_{R}\right)}{s}=\frac{\Delta n_{X}(t)}{s} \frac{\Gamma_{S_{1}}}{\Gamma_{S_{1}}+\Gamma_{S_{2}}}\left(\frac{t}{t_{2}}\right)^{2}\left(\frac{t_{2}}{t_{1}}\right)^{3 / 2},
$$

where $\tilde{t}_{R} \simeq t_{1}=\Gamma_{S_{1}}^{-1}$ and $t_{2}=\Gamma_{S_{2}}^{-1}$. Since we know from the discussion in the text that the $B$ asymmetry $Y_{B}$ can be expressed by using $\Delta n_{X}^{V}\left(\tilde{t}_{R}\right)$ as

$$
Y_{B} \equiv \frac{\Delta n_{B}}{s} \simeq \frac{\Delta n_{X}^{V}\left(\tilde{t}_{R}\right)}{s} f\left(N_{g}\right) \kappa
$$

we can obtain $Y_{B}$ in each case as follows:

$$
\begin{aligned}
& Y_{B} \simeq \Delta X \tilde{T}_{R} m_{3 / 2}^{\frac{4-n}{n-2}} M_{\mathrm{pl}}^{\frac{-2}{n-2}} f\left(N_{g}\right) \kappa \sin \delta \quad\left(\Gamma_{S_{1}}>\Gamma_{S_{2}}\right), \\
& Y_{B} \simeq \Delta X \tilde{T}_{R} m_{3 / 2}^{\frac{4-n}{n-2}} M_{\mathrm{pl}}^{\frac{-2}{n-2}}\left(\frac{\Gamma_{S_{1}}}{\Gamma_{S_{2}}}\right)^{1 / 2} f\left(N_{g}\right) \kappa \sin \delta \quad\left(\Gamma_{S_{1}}<\Gamma_{S_{2}}\right) .
\end{aligned}
$$

Although $Y_{B}$ has the same expression as that discussed in the text for the case of $\Gamma_{S_{1}}>$ $\Gamma_{S_{2}}$, there is an additional suppression factor $\left(\Gamma_{S_{1}} / \Gamma_{S_{2}}\right)^{1 / 2}$ for the case of $\Gamma_{S_{1}}<\Gamma_{S_{2}}$. Thus, if we impose this case to realize the observed $B$ asymmetry for the fixed values of $n, \kappa$ and $\sin \delta$, the higher reheating temperature $\tilde{T}_{R}$ is required in comparison with the case of $\Gamma_{S_{1}} \gtrsim \Gamma_{S_{2}}$.

On the other hand, the ratio of the energy density of the baryon and the dark matter can be expressed in both cases as

$$
\frac{\Omega_{B}}{\Omega_{\mathrm{DM}}} \simeq \frac{m_{p} Y_{B}}{m_{\mathrm{LP}} Y_{X}^{H}} \simeq f(3) \kappa \frac{m_{p}}{m_{\mathrm{LP}}} \frac{\Gamma_{S_{1}}}{\Gamma_{S_{2}}} .
$$


This suggests that the presently observed value of $\Omega_{B} / \Omega_{\mathrm{DM}}$ seems to be explained as far as $m_{\mathrm{LP}} \simeq\left(\Gamma_{S_{1}} / \Gamma_{S_{2}}\right) \kappa \mathrm{GeV}$ is satisfied. As far as we set up the hidden sector suitably, these lightest stable particles in the hidden sector are expected to behave as the required cold dark matter. 


\section{References}

[1] D. N. Spergei et al., Astrophys. J. Supl. Ser. 148 (2003) 175

[2] M. Tegmark el al., Phys. Rev. D69 (2004) 103501.

[3] H. P. Nilles, Phys. Rep. 110 (1984) 1; H. E. Haber and G. L. Kane, Phys. Rep. 117 (1985) 75; S. P. Martine, arXiv:hep-ph/9709356.

[4] S. M. Barr, R. S. Chivukula and E. Farhi, Phys. Lett. B241 (1990) 387; D. B. Kaplan, Phys. Rev. Lett. 68 (1992) 741.

[5] D. Hooper, J. March-Russell and S. M. West, Phys. Lett. B605 (2005) 228.

[6] M. Fujii and K. Hamaguchi, Phys. Lett. B525 (2002) 143; Phys. Rev. D66 (2002) 083501.

R. Allahverdi and M. Drees Phys. Rev. D70 (2004) 123522.

[7] L. E. Ibáñez and F. Quevedo, Phys. Lett. B283 (1992) 261.

[8] H. Dreiner and G. G. Ross, Nucl. Phys. B410 (1993) 188.

[9] D. Suematsu, J. Phys. G 31 (2005) 445.

[10] I. Affleck and M. Dine, Nucl. Phys. B249 (1985) 361.

[11] M. Dine, L. Randall and S. Thomas, Phys. Rev. Lett. 75 (1995) 398; M. Dine, L. Randall and S. Thomas, Nucl. Phys. B458 (1996) 291.

[12] R. J. Scherrer and M. S. Turner, Phys. Rev. D31 (1985) 681; R. Allahverdi, B. A. Campbell and J. Ellis, Nucl. Phys. B579 (2000) 355; A. Anisimov and M. Dine, Nucl. Phys. B619 (2001) 729.

[13] J. Ellis, A. Linde and D. Nanopoulos, Phys. Lett. B118 (1982) 59.

[14] V. A. Kuzmin, V. A. Rubakov and M. E. Shaposhnikov, Phys. Lett. B155 (1985) 36.

[15] S. Yu. Khlebnikov and M. E. Shapshnikov, Nucl. Phys. B308 (1988) 885; J. A. Harvey and M. S. Turner, Phys. Rev. D42 (1990) 3344. 
[16] W. Buchmüller and M. Plümacher, Int. J. Mod. Phys. A15 (2000) 5047, and references therein.

[17] Y. Suzuki, Super-Kamiokande Collaboration, Nucl. Phys. B (Proc. Suppl.) 91 (2001) 29; The SNO Collaboration, Phys. Rev. Lett. 87 (2001) 071301; Phys. Rev. Lett. 89 (2002) 011302; Phys. Rev. Lett. 89 (2002) 011301; The KamLAND Collaboration, Phys. Rev. Lett. 90 (2003) 021802; 92 (2004) 071301; hep-ex/0406035.

[18] Super-Kamiokande Collaboration, Y. Fukuda et at., Phys. Rev. Lett. 81 (1998) 1562; Phys. Lett. B436 (1998) 33; Phys. Lett. B433 (1998) 9; The K2K Collaboration, Phys. Rev. Lett. 90 (2003) 041801; Phys. Rev. Lett. 93 (2004) 051801.

[19] D. H. Oaknin and A. Zhitnitsky, Phys. Rev. D71 (2005) 023519.

[20] D. Suematsu, in preparation as KANAZAWA-05-09.

[21] D. Suematsu and Y. Yamagishi, Int. J. Mod. Phys. A10 (1995) 4521; M. Cvetič and P. Langacker, Phys. Rev. D54 (1996) 3570; D. Suematsu and G. Zoupanos, J. High Energy Phys. 06 (2001) 038.

[22] G. Cleaver, M. Cvetič, J. R. Espinosa, L. Everett and P. Langacker, Phys. Rev. D57 (1998) 2701.

[23] M. Fukugita and T. Yanagida, Phys. Lett. B174 (1986) 45; for a recent review, see for example, W. Buchmüller, hep-ph/0204288.

[24] H. Murayama, H. Suzuki, T. Yanagida and J. Yokoyama, Phys. Rev. Lett. 70 (1993) 1912; D. Suematsu and Y. Yamagishi, Mod. Phys. Lett. A38 (1995) 2923. 